\title{
Performance of Ground Penetrating Radar employing Buffer-Layered Antennas for Groundwater Detection based on Frequency Scaling Model
}

\author{
F. Farihas, I. Pasya
}

\begin{abstract}
Groundwater detection using classical electromagnetic (EM) wave ground penetrating radar (GPR), which utilizes single-input single-output (SISO) antenna configuration in open-air condition, results in poor signal propagation through soil. This paper proposes groundwater detection using enhanced GPR system utilizing buffer-layered antennas, where an additional medium is placed between the antenna and the ground, acting as a matching layer. The performance of the proposed scheme is investigated using frequency-domain time-domain (FDTD) based numerical simulation. Typically, numerical GPR simulation using real measurement parameters requires large number of meshing and therefore takes longer time to perform and is run on computers with high specification. In this paper, comparison between simulations using real scale and frequency scale model is also presented. Simulation result indicates improvement in wave propagation inside homogeneous soil using buffer-layered antenna, which can be observed in both real scale and frequency scale simulations.
\end{abstract}

Index Terms-Antenna, buffer-layered antenna, GPR, GprMax, ground penetrating radar, groundwater, frequency scaling model.

\section{INTRODUCTION}

G ROUNDWATER exploration activities have been actively promoted around the world to encourage new discoveries of water aquifers as people have become weary to depend solely on rainwater as their main water supply, especially in dry season. Groundwater detection techniques have developed from the ancient water divining and test drilling technique, to more current, non-destructive method such as seismic and ground penetrating radar (GPR).

GPR is an established non-invasive method which employs electromagnetic (EM) wave in subsurface sensing. Working on the principle of reflection and backscattering of EM waves,

This manuscript is submitted on $4^{\text {th }}$ April 2020 and accepted on $4^{\text {th }}$ June 2020.. Farihas was working with Panasonic Industrial Devices Malaysia before completing her MSc degree at University of Queensland, Australia in 2015. Currently she is pursuing her PhD degree at Universiti Teknologi MARA, Malaysia (e-mail: 2015383265@isiswa.uitm.edu.my).

I. Pasya is with Microwave Research Institute at Universiti Teknologi MARA, MALAYSIA (e-mail: idninpasya@gmail.com).

1985-5389/@ 2021 The Authors. Published by UiTM Press. This is an open access article under the CC BY-NC-ND license (http://creativecommons.org/ licenses/by-nc-nd/4.0/).
GPR is widely used to detect and image subsurface objects, typically at shallow depth in many applications such as civil engineering, archaeology, geology and forensic [1].

A classical GPR system for subsurface sensing carries out single-input single-output (SISO) transmission and utilizes a pair of antenna in an open-air condition [2]. It radiates EM wave into the ground via antenna and records EM waves reflected from boundaries of soils and other subsurface materials, if present. These reflections are contributed by the differences in electromagnetic properties of ground layers, particularly relative permittivity. More energy is reflected by abrupt boundaries compared to gradual boundaries that separate the contrasting mediums.

While GPR technology has developed rather progressively in the last three decades, in groundwater detection however, its performance is often limited by severe constraints as water aquifers are normally settled deep under the ground [3]. Paper [4] implies that water aquifers exist at far greater depth, as deep as $20 \mathrm{~m}$ beneath ground surface. In detecting groundwater, EM wave emitted from transmitting antenna experiences signal attenuation and reflection from different mediums and particles as it travels downward into soil. The rapid rates of signal attenuation, more than often result in reduced penetration depth of GPR in soils [5], which explains the average soil penetration depth by GPR is relatively at $5 \mathrm{~m}$ only [6-7].

In other research areas, for instance in submarine and medical device, usage and performance of antennas immersed in lossy medium have been investigated [8-10]. It turns out that, usage of intermediate medium in between mediums with contrast dielectric constants shows positive improvement in wave propagation characteristics, whereby, it smoothens out the transition of the radiating wave between the source and the scanning model and eventually increases radiation efficiency. Another study has discovered a possible relationship between transmitted power and thickness of intermediate medium, such that power changes periodically as thickness of the medium increases [11]. Unfortunately, the properties and structure of such intermediate medium have not been conclusively studied in these papers.

Carrying out an in-depth groundwater detection on actual 
ground field without any prior expectation may incur unnecessary cost. In most cases, initial simulations are often conducted and the results are used as references before performing real exploration on the field. Numerical modelling of GPR is based on FDTD method. Performing such simulation of low radio frequency for deep soil penetration using actual size model entails lengthy time and high in cost, in terms of software and computer's minimum specification. These challenges can be overcome by using frequency scaling model. Theoreticially, it is achievable as EM wave travels in terms of distance.

This paper presents GPR simulation of scanning radar scene models in detecting groundwater located $20 \mathrm{~m}$ under the ground, using a pair of antenna immersed in intermediate medium, hereafter termed as buffer. This paper also presents simulation results of same radar scene models, however, in a laboratory environment (lab scale radar scene) by employing frequency scaling model. Fig. 1 illustrates conventional GPR in SISO system, which uses antennas in open air condition, and the proposed enhanced GPR system which utilizes bufferlayered antenna.
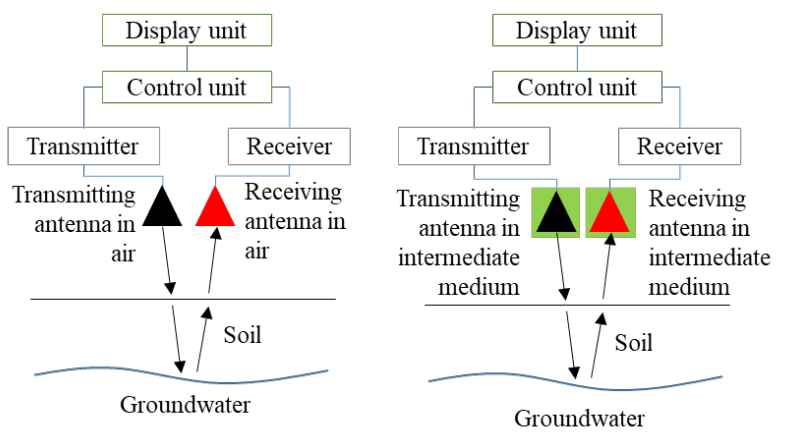

Fig. 1. Conventional GPR SISO system using air-antenna (left) and proposed GPR SISO system using buffer-layered antenna (right).

The work presented in this paper is organized as follows. Section II determines parameters of buffer layer and demonstrates simulation setups for GPR radar scenes. Section III discusses simulation results and section IV conculdes the paper's work.

\section{BUFFER LAYER PARAMETERS, SIMULATION RADAR SCENES AND BUFFER LAYERED ANTENNA}

There are three parts to this section: defining parameters of buffer, simulating the corresponding GPR performance in real scale and lab scale as proof of concept and lastly, designing a buffer-layered antenna for lab scale radar scene setup. CST Microwave Studio and GprMax are the two numerical modelling software involved in this study. GprMax is an open source software, which employs FDTD algorithm and is developed to perform GPR simulation and provide results in A and B scans [12]. Meanwhile, the commercial CST software is utilized to design buffer-layered antenna and simulate similar radar scene in terms of time signal.

\section{A. Parameters of Buffer}

In this study, dieclectric constant of the buffer, $\varepsilon_{r b}$, is calculated by taking the geometric average, that is the $n^{\text {th }}$ root of the product, of dielectric constant of every medium, $\varepsilon_{r i}$, that the electromagnetic wave signal intends to propragate through (1). This method is selected instead of using any random number, or the ordinary averaging method since it minimizes the effect of fluctuations of sampling, which in this case, is the number of propagating mediums [13]. Other properties, such as, conductivity and permeability are suggested to be equal to that of the soil.

$$
\varepsilon_{r b}=\sqrt[n]{\prod_{i=1}^{n} \varepsilon_{r_{i}}}
$$

Radar scene for this study involves two mediums, which are air and homogeneous soil as shown in Fig. 2. A 2-D real scale single-input single-output (SISO) radar scene is simulated using GprMax software, transmitting a $400 \mathrm{MHz}$ Ricker waveform from a point source antenna to obtain the optimum dimension (thickness $t$ ) of buffer layer while its width $d$, is set at halfwavelength. Half wavelength is the maximum antenna distance allowable to avoid multiple spectral peaks when using multipleinput multiple-output (MIMO) configuration, which is the final concept of the proposed system. However, this paper only discusses SISO configuration to prove the proposed concept.

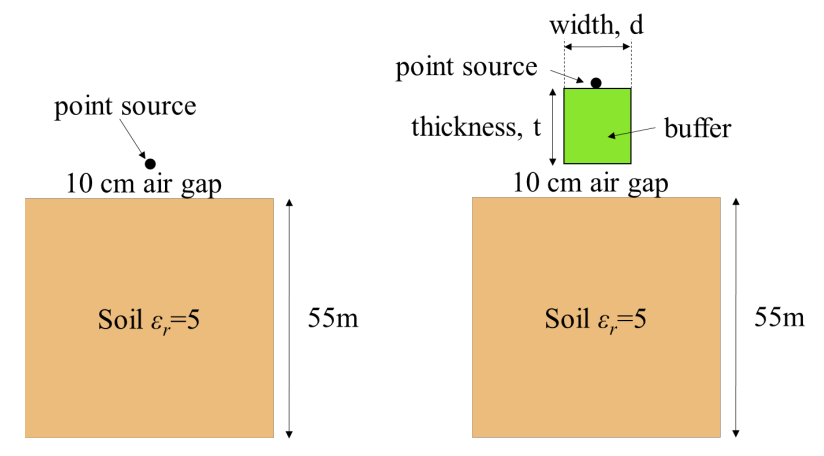

Fig. 2. 2D real scale radar scene in with signal source in air (left) and in buffer (right).

\section{B. Real Scale and Lab Scale Radar Scenes Simulation in GprMax}

Low radio frequency is naturally employed in GPR for groundwater detection since its long wavelength penerates deeper into the ground. In real scale radar scene, $400 \mathrm{MHz}$ wave is transmitted into $20 \mathrm{~m}$ deep of homogeneous ground field to simulate groundwater detection. To convert the radar scene into a practical depth for lab measurement purposes, for instance 1 m depth, wavelength of the $400 \mathrm{MHz}$ wave $(75 \mathrm{~cm})$ is divided by an integer, 20 resulting to $37.5 \mathrm{~mm}$, which is the equivalent wavelength of an $8 \mathrm{GHz}$ waveform. Correspondingly, other dimensions of the radar scene are also scaled down by the same factor, 20. Figs. 3 and 4 present the real and lab scale radar scenes for GprMax simulation. A $400 \mathrm{MHz}$ and an $8 \mathrm{GHz}$ Ricker waveform are used to simulate two conditions in each respective radar scene: when point source is in air, and when point source is in buffer layer medium. 


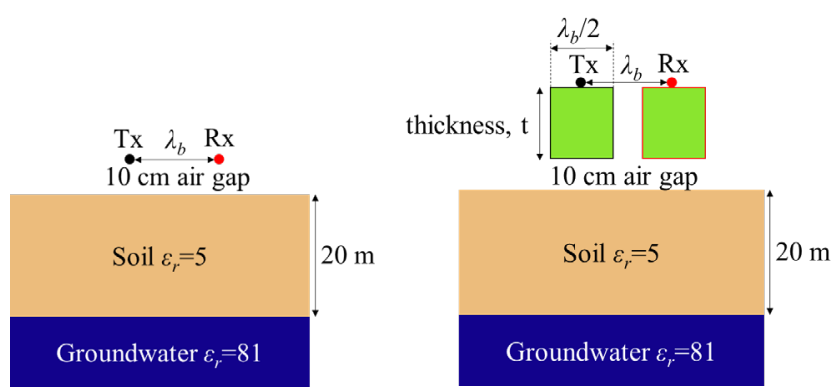

Fig. 3. Real scale radar scenes using $400 \mathrm{MHz}$ signal source, tranmissted in air (left) and in buffer (right).
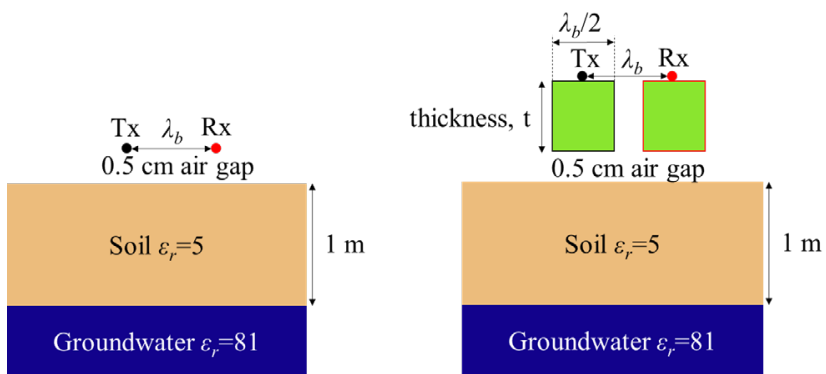

Fig. 4. Lab scale radar scenes using $8 \mathrm{GHz}$ signal source, transmitted in air (left) and in buffer (right).

\section{Buffer-layered Vivaldi Antenna in Lab Scale Radar Scene}

Following the prood of concept simulation in part B, a 3D simulation using buffer-layered antenna detecting the presence of groundwater based on SISO system in lab scale measurement is performed using CST software. GPR system typically uses horn antennas to transmit and receive EM waves as they are best known to provide high gain transmission and minimum loss when receiving EM signals. However, following the concept of this study, which at later stage will be applying the notion of $3 \times 3$ MIMO system, horn antennas are not particularly suitable to be used due to their relatively large size.

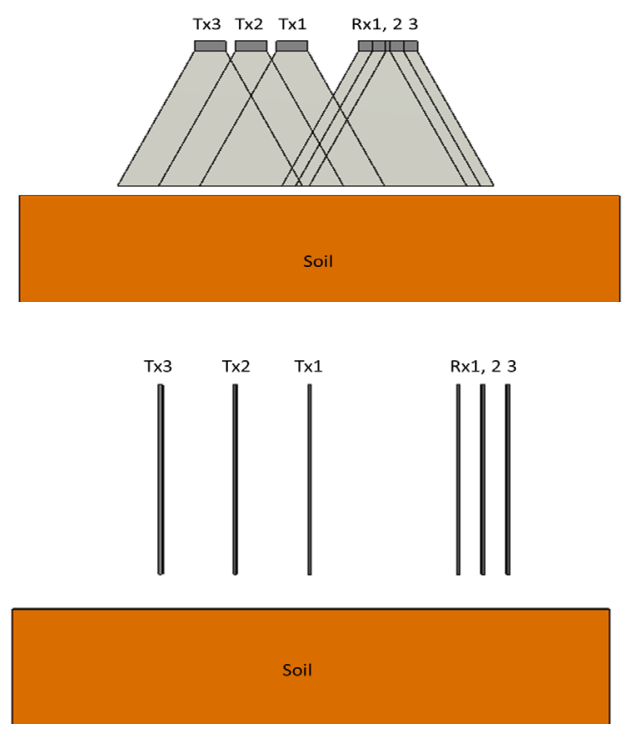

Fig. 5. Radar scene in MIMO system using horn antenna (top) and vivaldi antenna (bottom) for $8 \mathrm{GHz}$ signal transmission.
In MIMO, antenna receivers are placed at half-wavelength intervals while antenna transmitters are separated at three times of that to achieve space diversity [14]. The size of horn antennas for $8 \mathrm{GHz}$ transmission, on the other hand, exceeds its halfwavelength. Hence, placing buffer-layered horn antenna physically next to each other for a $3 \times 3$ MIMO system is not feasible as demonstrated in Fig. 5. Instead, vivaldi antenna is designed and applied in the radar scene.

Vivaldi antenna is generally conceived as the planar form of horn antenna as it has similar contour as ridged horn antenna and it outperforms other planar type antennas, particularly in terms of gain. An exponential tapered slot edge with miscrostrip feed structure, coplanar vivaldi antenna is employed for $8 \mathrm{GHz}$ transmission. The tapered slot is designed using standard exponential equation as in (2), where $r$ is the opening rate of the exponential taper, and $a$ is a constant.

$$
y=a e^{-r t}
$$

Fig. 6 presents the design architecture of vivaldi antenna used in this study. The structure of vivaldi antenna works over wide frequency range and has the capability to extend the low-end bandwidth limit and provide enhanced antenna matching [15]. The performance of the designed antenna as transmitter and receiver in the lab scale groundwater detection radar scene is simulated using SISO system in two conditions such that, when it is in air and when it is layered with buffer medium as illustrated in Fig. 7. The simulations are performed using CST simulation software.
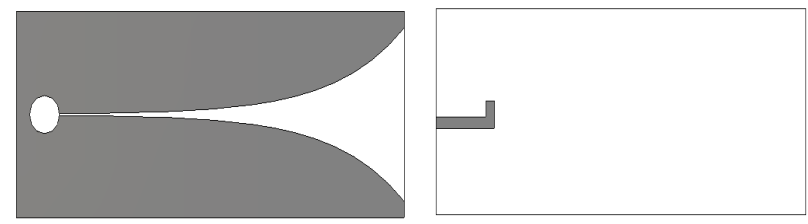

Fig. 6. Vivaldi antenna in front view (left) and its feeding line in back view (right).
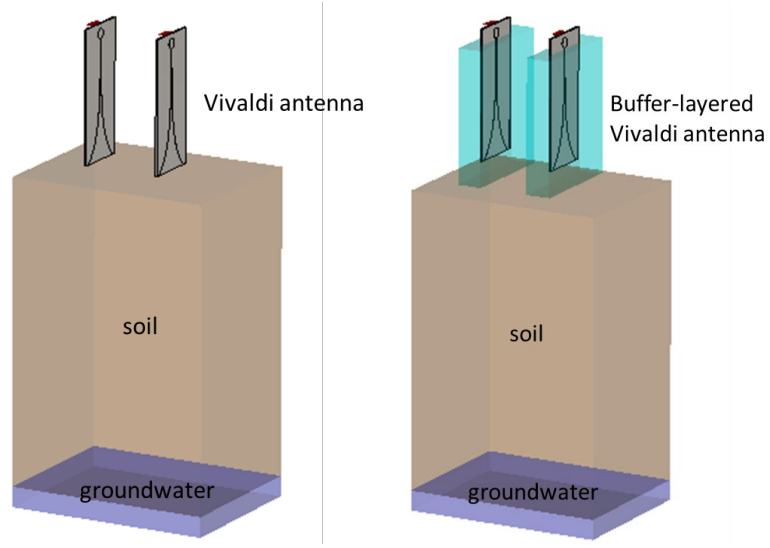

Fig. 7. CST simulation of lab scale radar scene using vivaldi antenna in air (left) and in buffer layer (right). 


\section{Simulation RESUlt AND Discussion}

Simulation scanning result by GprMax are presented in terms of A-scan and B-scan. A-scan relects 1 dimensional result of signal travelling in time, while B-scan portrays 2 dimensional model of the radar scene.

In secion II part A of defining parameters of buffer, based on Fig. 1(a) and following equation (1), dielectric constant of buffer is calculated to be 2.23 after taking the square root of the product of dielectric constant of air and homogeneous soil. Fig. 8 yields that EM wave travels longer in homogeneous soil when it is emitted from a buffer-layered transmitter, compared to when the transmitter is in air. The reduction in attenuation rate could possibly be initiated by the extension of skin depth as difference in dielectric constant between soil and air is reduced when buffer is introduced as intermediate medium between the two.

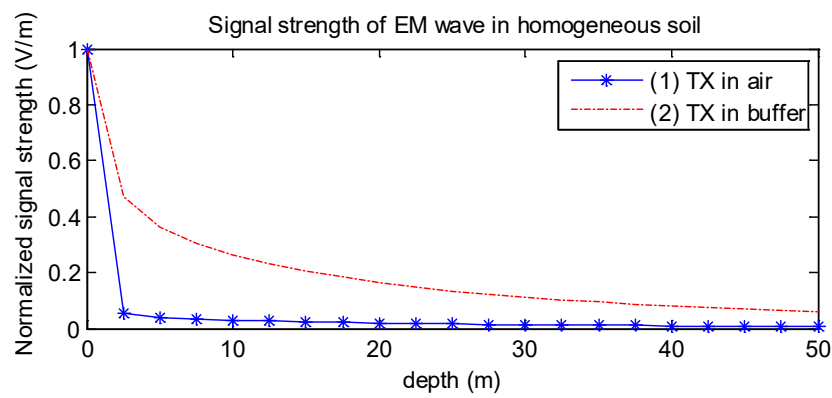

Fig. 8. Normalized signal strength in homogeneous soil when EM wave is emitted using normal and buffer-layered transmitter.

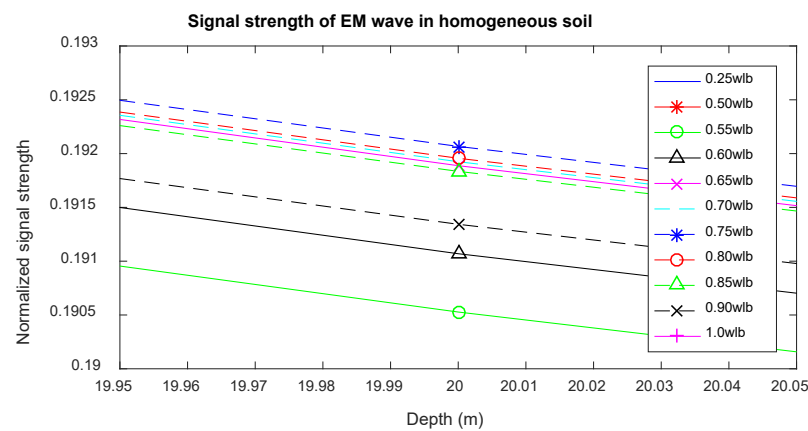

Fig. 9. Normalized signal strength of EM wave in homogeneous soil, transmitted from buffer-layered transmitter, of different buffer layer thickness.

In narrowing down the dimension of buffer layer, Fig. 9 exhibits the normalized signal strength of EM wave as it propagates down into the soil from transmitter immersed in various thickness of buffer layer. It is observed that signal strength is not directly proportional to the thickness of buffer layer. Normalized signal strength rises and reaches its peak before declining down as the buffer layer thickness continues to be increased to a full wavelength. This pattern of dependency is similar to that found in [11]. At $20 \mathrm{~m}$ depth of homogeneous soil, normalized signal strength is highest when the wave is emitted from buffer-layered transmitter, which thickness of the buffer layer equals to three quarter wavelength.
Based on the results obtained in section II part A, proof of concept in real and lab scale radar scenes as illustrated in Figs. 3 and 4 are simulated using GprMax, and the simulation results are presented in the following figures. A-scans in Figs. $10-12$ show recordings of signals received by the receiver as EM wave signal travels from point source into the homogeneous soil and finally meeting the flat surface of groundwater. The first recorded spike (at about $10 \mathrm{~ns}$ for real scale and $0.5 \mathrm{~ns}$ for lab scale) is called direct wave, which translates to the wave dispersed within the moment it exits the point source. There is barely significant differences in direct wave between real and lab scale models. The other visible spike (at time around $31 \mathrm{~ns}$ for real cale and $1.6 \mathrm{~ns}$ for lab scale) is the reflected wave from when the transmitted signal meets the flat surface of groundwater. Reflected signals in lab scale radar scene are observed to have more distinguished ripples compared to the ones recorded in real scale radar scene. This is most likely the result of differences in sampling frequencies used in both models.
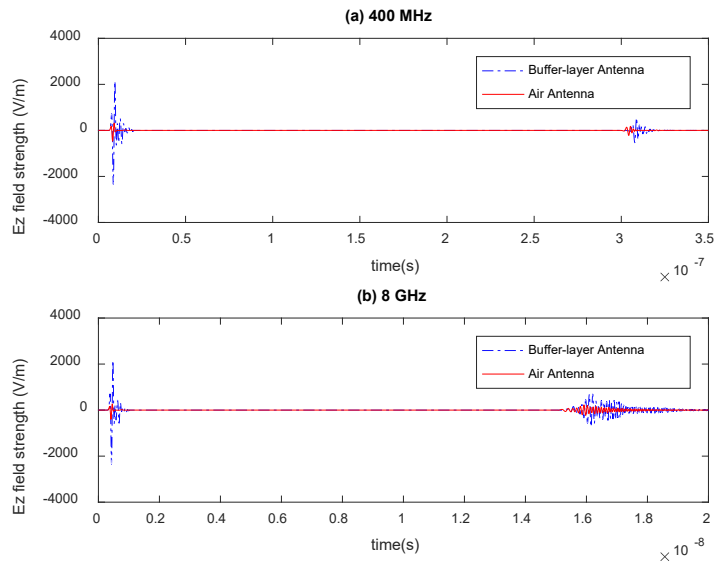

Fig. 10. Time domain $E_{z}$ electric field strength of groundwater incident using buffer-layered antenna and air-antenna in; (a) real-scale using $400 \mathrm{MHz}$; (b) lab-scale using $8 \mathrm{GHz}$.
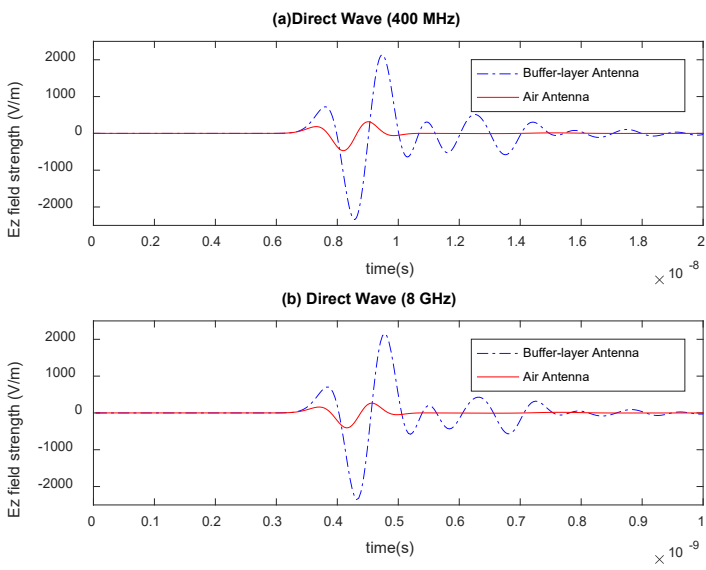

Fig. 11. Signal strength of direct wave using buffer-layered antenna and airantenna in; (a) real-scale using $400 \mathrm{MHz}$; (b) lab-scale using $8 \mathrm{GHz}$. 

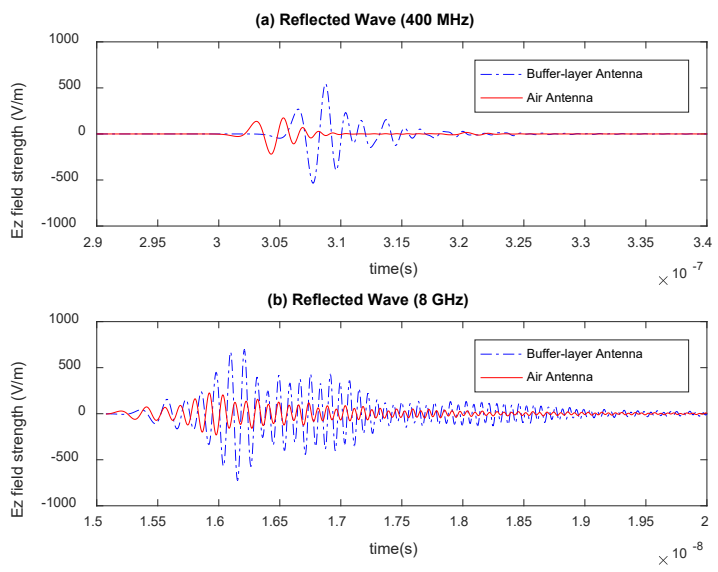

Fig. 12. Signal strength of reflected wave from groundwater incident using buffer-layered antenna and air-antenna in; (a) real-scale using $400 \mathrm{MHz}$; (b) lab-scale using $8 \mathrm{GHz}$.

In both radar scenes, the receiver in buffer-layered antenna records much elevated direct wave and relatively stronger signal strength of reflected wave from groundwater compared to the receiver in air-antenna setup. Table 1 shows the maximum level of signal strength, in unit $\mathrm{V} / \mathrm{m}$, of each direct

TABLE I

SIGNAL STRENGTH RECORDED By RECEIVER

\begin{tabular}{llcc}
\hline \hline $\begin{array}{l}\text { Radar Scene } \\
\text { (Frequency) }\end{array}$ & Antenna in & $\begin{array}{c}\text { Real Scale } \\
(400 \mathrm{MHz})\end{array}$ & $\begin{array}{c}\text { Lab Scale } \\
(8 \mathrm{GHz})\end{array}$ \\
\hline Direct & Buffer & $2.1332 \times 10^{3}$ & $2.1586 \times 10^{3}$ \\
Wave & Air & $0.3156 \times 10^{3}$ & $0.2673 \times 10^{3}$ \\
& & & \\
Reflected & Buffer & $0.5463 \times 10^{3}$ & $0.6964 \times 10^{3}$ \\
Wave & Air & $0.1742 \times 10^{3}$ & $0.225 \times 10^{3}$ \\
& & & \\
SS RW/DW & Buffer & $25.6 \%$ & $32.3 \%$ \\
& Air & $55.2 \%$ & $84.2 \%$
\end{tabular}

Unit for signal strength is $\mathrm{V} / \mathrm{m}$

and reflected waves recorded by receivers in both antenna setups for real and lab scale radar scenes. In lab scale, the signal strength of reflected wave from surface of groundwater is recorded at $225.0 \mathrm{~V} / \mathrm{m}$ and $696.4 \mathrm{~V} / \mathrm{m}$ using air-antenna and buffer-layered antenna, respectively. In real scale, the corresponding figures are $174.2 \mathrm{~V} / \mathrm{m}$ and $546.3 \mathrm{~V} / \mathrm{m}$. Higher reflected wave signal strength suggests lesser signal lost during penetration and travelling through the homogeneous soil medium, hence provides higher possibility of detecting and locating the groundwater. The differences in reflected signal strength between air-antenna and buffer-layered antenna in both scales are relatively similar, which are $29.2 \%$ in real scale and $27.5 \%$ in lab scale. This implies that simulation employing frequency scaling model gives comparable results.

B-scans in Figs. 13 and 14 demonstrate that reflection of groundwater can be seen more prominently in buffer-layered antenna system, albeit a slight delay in the response indicating small discrepancies in depth of groundwater, at about $20.5 \mathrm{~m}$ under the ground for real scale, and $1.05 \mathrm{~m}$ for lab-scale. The cause for delay in response is possibly related to velocity changes of the signal wave as it enters mediums with different refractive indexes. In this study, where the depth of soil is known, such circumstances can be corrected with mathematical algorithm at later stage.
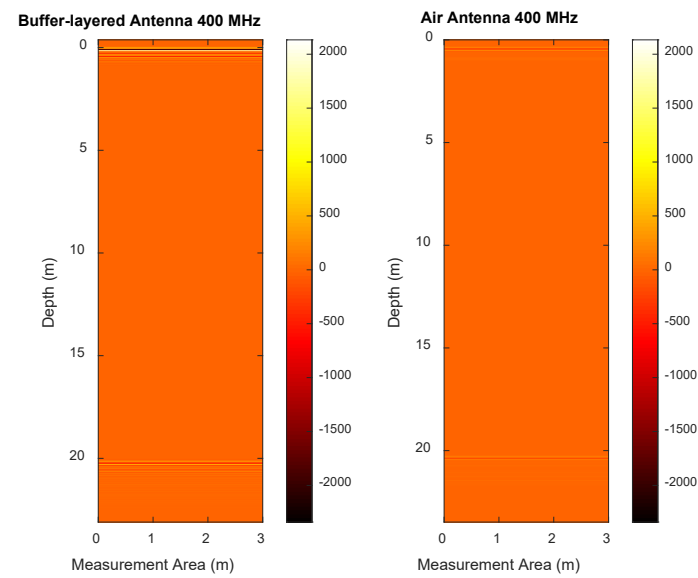

Fig. 13. B-scan of real scale radar scene using buffer-layered antenna (left) and air antenna (right).
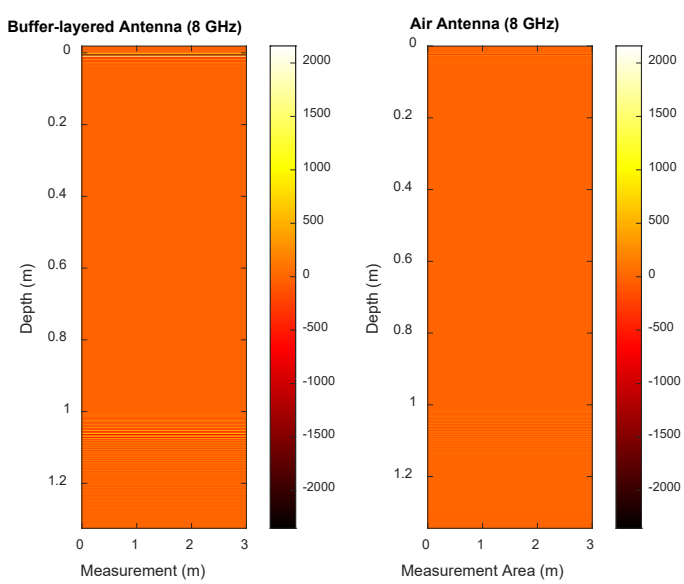

Fig. 14. B-scan of lab-scale radar scene using buffer-layered antenna (left) and air antenna (right).
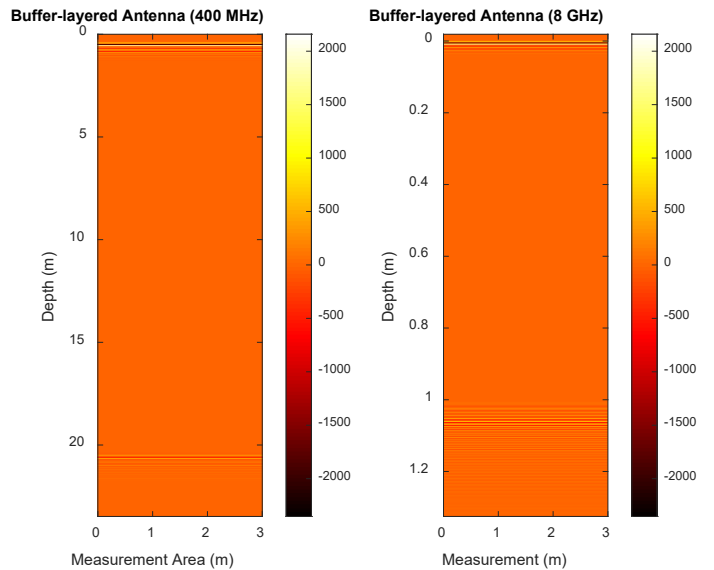

Fig. 15. Comparison of grounwater reflection using buffer-layared antenna in real-scale (left) and lab-scale (right). 
Fig. 15 compares detection level of groundwater when the enhanced GPR system with buffer-layered antenna is simulated in real scale and lab scale. Given the same equivalent radar scene, depth of soil and simulation setting, GprMax simulation in lab scale generates similar result as simulation in real scale. Simulation time is greatly reduced by $95 \%$ from $900 \mathrm{~s}$ to $45 \mathrm{~s}$, and memory usage is lessened by $87 \%$ in lab scale simulation.

TABLE II

GPRMAX SIMULATION DATA

\begin{tabular}{cccc}
\hline \hline $\begin{array}{c}\text { Measurement } \\
\text { Scale }\end{array}$ & Frequency & $\begin{array}{c}\text { Simulation } \\
\text { Time }\end{array}$ & $\begin{array}{c}\text { Memory } \\
\text { Usage }\end{array}$ \\
\hline Real scale & $400 \mathrm{MHz}$ & $900 \mathrm{~s}$ & $903 \mathrm{MB}$ \\
Lab scale & $8 \mathrm{GHz}$ & $45 \mathrm{~s}$ & $116 \mathrm{MB}$ \\
& & & \\
\hline \hline
\end{tabular}

\section{CONCLUSION}

In this paper, investigation of improving deep groundwater detection using buffer-layered antenna is presented through numerical simulations in real scale and frequency scale models. Applying buffer-layered antenna, comprising of structure which includes an additional intermediate medium layer between the antennas and ground, further enhances transmitting signal propagation disctance, and the receiving signal power. This provides an imporoved performance of groundwater detection at $20 \mathrm{~m}$ beneath homogeneous soil, compared to utilization of conventional antennas with similar base antenna properties. Simulating the radar scene in a laboratory environment by employing frequency scaling model, produces similar result as in real scale measurement in shorter amount of simulation time and lesser computer memory usage.

\section{ACKNOWLEDGEMENT}

The authors would like to thank Universiti Technology MARA for their financial support under Geran Inisiatif Penyelidikan (GIP) grant 600-IRMI/GIP 5/3 (0011/2016).

\section{REFERENCES}

[1] A. S. Turk, A. K. Hocaoglu, and A. A. Vertiy, Subsurface Sensing. USA: New Jersey, 2011 pp. 7-83.

[2] D. H. N. Marpaung and Y. Lu, "Investigation of MIMO and SAR Performance on Several Applications of UWB GPR", pp. 196-199, 2014.

[3] R. Kirsch, "Groundwater Geophysics: A Tool for Hydrogeology," Environmental and Engineering Geoscience, vol. 17, no. 1, 2011.

[4] R. Frink, "Preserving Trans-Boundary Aquifers: A Precious Resource for Our Future Generations," Pacific McGeorge Glob. Bus. Dev. Law Journal, vol. 26, no. 2, pp. 503-530, 2013.

[5] M. R. Mahmoudzadeh, A P. Frances, M. Lubczynski, and S. Lambot, "Using Ground Penetrating Radar to Investigate the Water Table Depth in Weathered Granites - Sardon Case Study, Spain," J. Appl. Geophys., vol. 79, pp. 17-26, 2012.

[6] Z. Zhu, X. He, G. Lu, Q. Liu, and J. Li, "Ground Penetrating Radar Exploration for Ground Water and Contamination," Piers, pp. 1316-1320, 2009.

[7] H. Liu and M. Sato, "Dynamic Groundwater Level Estimation by the Velocity Spectrum Analysis of GPR," $201214^{\text {th }}$ Intl. Conf. Gr. Penetrating Radar, pp. 413-418, 2012.

[8] S. Lambot and F. Andre, "Full-Wave Modelling of Near-Field Radar Data for Planar Layered Media Construction," IEEE Trans. Geoscience and Remote Sensing, vol. 52, no. 5, pp. 2295-2303, 2012.
[9] L. Gao et al., "Computing the Plane Electromagnetic Wave Transient Propagation in Lossy Medium Based On COMSOL," in 2015 Int. Conf. on Estimation, Detection and Information Fusion, pp. 367-371, 2015.

[10] H. Yoshida, N. Iwakiri, T. Fukuda, M. Deguchi, and S. Onogi, "Measurements of Underwater Electromagnetic Wave Propagation," IEEE Underwater Technology, pp. 1-5, 2015.

[11] S. Park et al, "A New Receiver Antenna with Buffer Layer for Wireless Capsule Endoscopy in Human Body," Intl. Symposium on Antennas and Propagation, pp. 1-4, 2008.

[12] A. Giannopoulos, "Modeling Ground Penetrating Radar by GprMax," Construction and Building Material, vol. 19, no. 10, pp. 755-764, 2005.

[13] D. Clark-Carter, "Geometric Mean," in Encyclopedia of Statistics in Behavioral Science, John Wiley \& Sons, Ltd, 2005. DOI: 10.1002/0470013192.bsa376.

[14] A.M. Haimovich, R.S. Blum, and L. J. Cimini, "MIMO Radar with Widely Separated Antennas," IEEE Signal Process. Mag., vol. 25, no. 1, pp. 116-129, 2008.

[15] Z. Tahar, D. Xaviwe, and M. Benslama, "An Ultra-Wideband Modified Vivaldi Antenna Applied to Ground and Through the Wall Imaging," Progress in Electromagnetics Research C, vol. 86, no. August, pp. 111122, 2018.

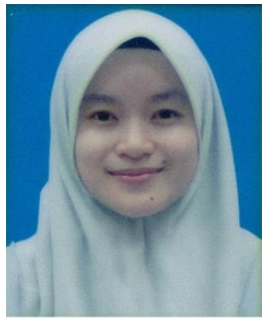

F. Farihas received her B.S. degree in electrical engineering from Purdue University, Indiana, USA in 2003 and the M.S. degree in electrical engineering from University of Queensland, Australia in 2015. She is currently pursuing the Ph.D degree in electrical engineering at Univerisiti Teknologi MARA, Malaysia. From 2004 to 2013, she was an RF design engineer with the Research and Development Business Center of Panasonic Industrial Devices, Malaysia. Ms. Farihas was a recipient of the Student Paper Award in 2017 Malaysia-Japan Workshop on Radio Technology by the IEICE.

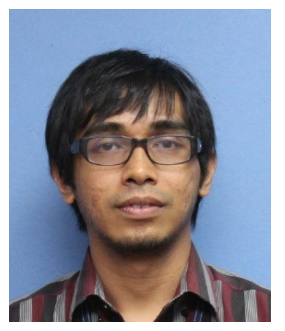

Dr. Idnin Pasya is a Senior Lecturer in the Faculty of Electrical Engineering, Universiti Teknologi MARA. He received the B. E. and M. E. degrees in Information and Communication Engineering from Tokyo Denki University in 2004 and 2006, respectively, and his Ph.D. in Communication Engineering from the same university in 2015. Previously, he worked as an Engineer in Toshiba PC \& Network, Tokyo, Japan, from 2006 to 2009, and Pradonet Technology Sdn. Bhd. in 2009 (Senior Eng.), before joining UiTM in the same year. He became an active Member of IEEE since 2010, where he contributed to many research publications in the area of antennas and propagation, ultra wideband communication systems and devices, and MIMO radar and its applications. Dr. Idnin received the IEEE MTT Best Paper Award in 2014 IEEE Radio and Wireless Symposium, held in California, USA. He is currently a member and Deputy Director of Microwave Research Institute (MRI), UiTM. He contributed to IEEE as an executive committee for IEEE AP/MTT/EMC Joint Chapter for the year 2017-2020. He is also an active member of SIRIM Technical Committee on "Electromagnetic Field" (TC/E/6) for the year 2016-2019, and 2020. Dr. Idnin served as the head of Radar in a non-profit organization, Malaysia Radar and Navigation (MyRAN) group, for the year 2018-2019. 